\title{
APPLICATIONS OF AUTOMATA THEORY AND ALGEBRA
}

Via the Mathematical Theory of Complexity to Biology, Physics, Psychology, Philosophy, and Games 


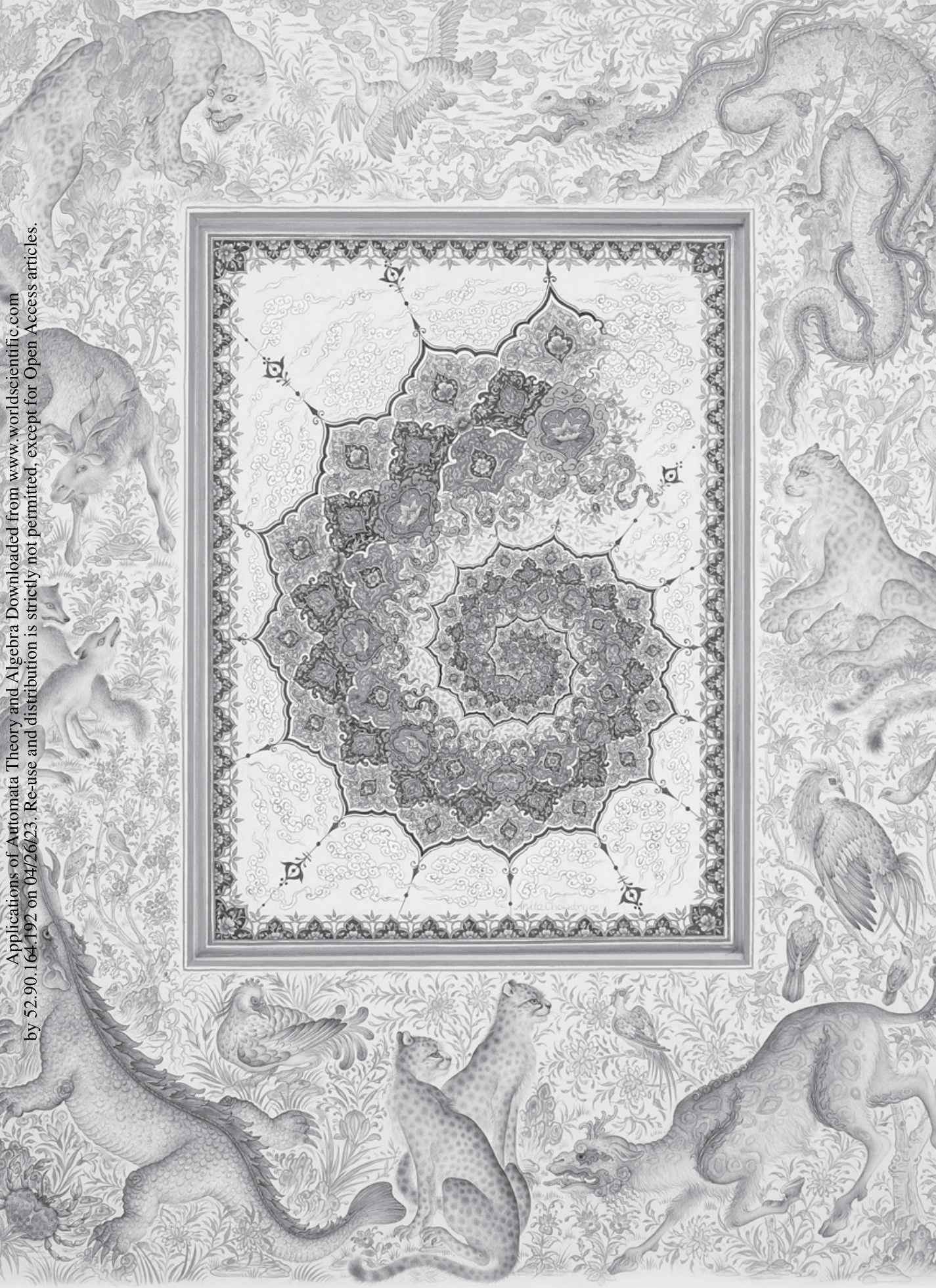




\section{APPLLCATIONS OF}

\section{AUTOMATA THEORYY AND ALGEBRA} Via the Mathematical Theory of Complexity to Biology, Physics, Psychology, Philosophy, and Games

\section{John Rhodes}

University of California at Berkeley, USA

Edited by

Chrystopher L. Nehaniv

University of Hertfordshire, UK

Foreword by

Morris W. Hirsch

University of California at Berkeley, USA 
Published by

World Scientific Publishing Co. Pte. Ltd.

5 Toh Tuck Link, Singapore 596224

USA office: 27 Warren Street, Suite 401-402, Hackensack, NJ 07601

UK office: 57 Shelton Street, Covent Garden, London WC2H 9HE

\title{
British Library Cataloguing-in-Publication Data
}

A catalogue record for this book is available from the British Library.

Front cover image: "Illuminated Dragons" by Anita Chowdry, collection of Najma Kazi.

Back cover image and page (ii): "Nautilus" by Anita Chowdry, collection of Lionel de Rothschild.

\begin{abstract}
APPLICATIONS OF AUTOMATA THEORY AND ALGEBRA Via the Mathematical Theory of Complexity to Biology, Physics, Psychology, Philosophy, and Games

Copyright (C) 2010 by World Scientific Publishing Co. Pte. Ltd.

All rights reserved. This book, or parts thereof, may not be reproduced in any form or by any means, electronic or mechanical, including photocopying, recording or any information storage and retrieval system now known or to be invented, without written permission from the Publisher.
\end{abstract}

For photocopying of material in this volume, please pay a copying fee to the Copyright Clearance Center, Inc., 222 Rosewood Drive, Danvers, MA 01923, USA. In this case permission to photocopy is not required from the publisher.

ISBN-13 978-981-283-696-0

ISBN-10 981-283-696-9

ISBN-13 978-981-283-697-7 (pbk)

ISBN-10 981-283-697-7 (pbk)

Printed in Singapore. 


\section{Contents}

Foreword to Rhodes' Applications of Automata Theory and Algebra vii Morris W. Hirsch

Editorial Preface

Chrystopher L. Nehaniv

xiii

Prologue: Birth, Death, Time, Space, Existence, Understanding, Science, and Religion

John Rhodes

1. Introduction

2. What is Finite Group Theory? 9

Bibliography . . . . . . . . . . . . . . . . . 14

3. A Generalization of Finite Group Theory to Finite Semigroups 15 Bibliography . . . . . . . . . . . . . . . 33

4. A Reformulation of Physics 35

Bibliography ...................... 54

5. Automata Models and the Complexity of Finite State Machines 55

Part I. The Prime Decomposition Theorem . . . . . . . . . . . 55

Part II. Complexity of Finite State Machines . . . . . . . . . . . 67

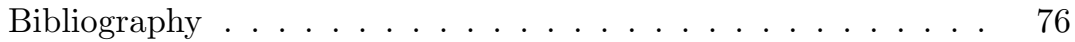

Appendix to Chapter $5 \ldots \ldots \ldots \ldots$. . . . . . . . . 77 
Bibliography . . . . . . . . . . . . . . . . . . 108

6. Applications 111

Introduction . . . . . . . . . . . . . . . . . . . 111

Part I. Analysis and Classification of Biochemical Reactions . . 114

Bibliography . . . . . . . . . . . . . . 175

Part II. Complexity of Evolved Organisms _ . . . . . . . . . 176

Appendix to Part II . . . . . . . . . . . . . . . . . . . . . . 195

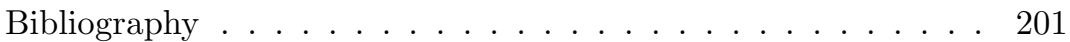

Part III. The Lagrangian of Life . . . . . . . . . . . . . . . . 203

A. The Laws of Growing and Evolving Organisms . . . . 203

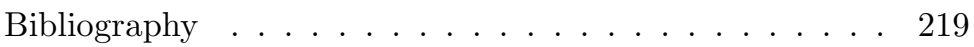

B. Complexity, Emotion, Neurosis and Schizophrenia . . . 220

Bibliography . . . . . . . . . . . . . 239

Part IV. Complexity of Games . . . . . . . . . . . . . . 241

Bibliography . . . . . . . . . . . . . . 256

$\begin{array}{ll}\text { Index } & 257\end{array}$ 


\section{Foreword to Rhodes' Applications of Automata Theory and Algebra}

John Rhodes came to Berkeley in 1963 as assistant professor of mathematics and has been here ever since. In addition to full time teaching and research, he has been a real estate entrepreneur (I made a little money with him), and a therapist, and campus activist. In the sixties he and Krohn ran their own consulting firm, which supported their graduate students and ran conferences. Eventually it was sold at a profit.

I recall vividly my enchantment upon first seeing, in 1969, the mimeographed notes that are almost identical with this book. I knew nothing of the algebra and automata theory behind it, and what little I know I've learned from John. The book is still strikingly original, but in those days it was astonishing. Computers were practically unknown, not only to the public but to the vast majority of scientists, mathematicians and engineers. Many subjects intimately connected to the book were likewise either nonexistent or known only to very small groups: Complexity Theory, Neural Networks, Cellular Automata, Catastrophe Theory, Chaotic Dynamics, Genetic Algorithms .... Pure mathematics was just beginning to come out of a period of fruitful but esoteric rigorous axiomatic development, typified by Bourbaki. Applied mathematics, science and engineering had advanced sufficiently that it could begin to appreciate and use some of the more abstruse mathematical theories.

Rhodes' 1962 doctoral thesis at MIT was not only greatly original in its content, but also in the fact that it was a joint work with Kenneth Krohn. When the outside examiner at Rhodes' thesis defense complained that a Harvard student had written the same thesis, there was no little consternation. Rhodes and his coauthor Krohn had in fact informed their supervisors of their collaboration, saying "It is worth four theses but we 
only want two". When Krohn handed in his thesis he merely crossed out Rhodes' name and typed in his own.

In his thesis Rhodes (with Krohn) looked at semigroups as finite state machines. Mathematically there is a complete equivalence, but the perspective is different in the two approaches. To tinker with the multiplication table of a group or a semigroup is still a rather strange idea to an algebraist; but nothing could be more natural than to adjust the workings of a machine. Certain constructions are difficult to handle algebraically, but correspond to simple operations on machines. The wreath product of two semigroups, for example, translates into the cascade of two machines, where the output of the first is fed as input into the second.

On the other hand, the algebraic viewpoint unifies many subjects in which a machine approach seems at first sight to be inappropriate, such as the topics covered in this book. Anything with states, inputs and outputs can be usefully looked at in semigroup terms.

The Krohn-Rhodes theory represents any finite semigroup $S$ as the homomorphic image of a subsemigroup of the wreath product of a finite sequence of semigroups acting on finite sets, in which permutation groups and combinatorial semigroups (of noninjective maps) alternate, beginning and ending with combinatorial semigroups. The minimal number of groups in such a representation is the complexity $\theta(S)$ of the semigroup $S$.

The complexity of a finite state machine is the minimal number of reversible computations which, in any cascade representation, must be performed successively, separated by a irreversible redirection of output. Here the reversible computations are done by groups, and the irreversible ones by combinatorial semigroups.

Rhodes outlines the rich and elegant axiomatic theory of complexity in the first chapters. What does it have to do with Biology, Physics, Psychology and Games?

The basic philosophical outlook in the rest of the book is this: Any system which can be investigated scientifically can be - or perhaps must be - usefully approximated as a finite state machine. To take only some easy cases: A game such as chess has only finitely many positions. The state of an organism is describable by finitely many chemicals, whose concentrations can be approximated by a finite set of vectors. These concentrations change in response to inputs from the environment, which can be similarly approximated.

Rhodes argues that many scientific and philosophical questions can be formulated in terms of the complexity of suitable semigroups, and that to 
do so leads to interesting theories and conjectures. Often these are based on computations of complexity whose outcomes are by no means obvious.

The simplest application is to the Theory of Games. The complexity of a game is defined as the minimum complexity of machines which can play the game perfectly (e.g., so as to achieve the von Neumann value of the game). Rhodes proposes that the rules of board games have evolved so as to maximize complexity. Rough estimates lead him to conjecture that this maximum is of the order of the typical number of moves in a game between expert players. For $7 \times 7 \mathrm{Hex}$, he proves that the complexity is no greater than 24. For Go he proves it is less than 200. Rhodes conjectures that the complexity of Go and Hex, but not of 3-dimensional Tic-Tac-Toe, tends to infinity with the size of the board.

Rhodes looks closely at a semigroup $S$ representing the classical Krebs cycle in Biochemistry. Here he argues that it is not just the complexity that is significant, but also the maximal prime factor groups of subgroups of $S$. In a tour de force he calculates that these are just $\mathbb{Z}_{2}$ and $\mathbb{Z}_{3}$, that the complexity is 2 .

More complex chemical cycles lead to other prime groups - prime order cyclic groups and Simple Nonabelian Groups (SNAGs). Rhodes considers the oxidative pentose phosphate cycle, and suggests that highly transitive permutation groups, close to SNAGs, appear. He points out that the computation cannot be carried out owing to the failure of the Principle of Superposition: that all reactions that occur are derived from the basic ones by independent summation. He proposes that "the groups which actually do appear are large subgroups of the alternating or symmetric groups which theoretically appear under the superposition principle." The latter groups include the 4-transitive Mathieu group on 12 letters. These SNAGs "measure the intricacy of the interconnection of the reactions."

These computations are based on a labeled directed graph derived from the chemistry. Similar analyses can be done, Rhodes suggests, on flow charts of computer programs, or on graphs arising from the configurations in Conway's Game of Life. He asks the interesting question, "How does the complexity of the configurations change with time?"

In Evolution the application of semigroup theory is necessarily more speculative, but also more comprehensible. Here the objective is not precise computations of complexity or SNAGs, but rather general principles influencing Evolution.

Highly evolved organisms, he suggests, are in "perfect harmony" with their environments - otherwise they would either die out or evolve further: 
Either the organism uses effectively more of the possibilities, or the possible configurations diminish with lack of use.

Thus if an organism has stopped evolving (e.g., the cell) then it must be stable under the forces of evolution and thus the complexity must be close to the possible number of configurations.

Rhodes then derives the following highly nontrivial and surprising conclusions from this principle:

- Enzymes catalyze (in almost all cases) specific reactions.

- A Mendelian genetic theory must hold for all evolved organisms.

- No simplification of the basic (but immense) data of an evolved organism is possible.

- Evolved organisms have internal relations of great depth.

- In the cell, the control of the genes over metabolism need not be absolutely complete but must be very good.

Rhodes discusses R. Thom's controversial models of morphogenesis. These are at the opposite end of the mathematical spectrum, based as they are on continuous state spaces and differential equations. Yet Thom's approach is based on the finite number of elementary catastrophes. Moreover Rhodes, like Thom, assumes that biological development maximizes some potential-like function on the state space.

Rhodes points out what many biologists consider a glaring weakness in Thom's theory: it offers no way to identify physical variables with the potential function. (Thom would no doubt say that that is a task for biologists.) Rhodes claims his principle of evolution can be of service here, as the possible elementary catastrophes will be intimately related to the states of the machine representing the organism. Rhodes suggests that a certain generalized Lagrangian function that he describes

is the function that all living organisms are attempting to maximize. But its definition requires modeling organisms with finite state machines plus a lot of algebra ... So we will need Thom's detailed results extended to arbitrary diffeomorphisms ... plus detailed modeling of organisms by finite state machines, plus algebraic theory of complexity ...

One interesting thing appears on both the finite side and on the differentiable side, namely Lie groups ... most (all?) SNAGs come from Lie algebras (plus some 'twists') via the Chevalley method.

Recently the theory of 'punctuated equilbrium' has received a good deal 
of attention, especially in the popular writings of Stephen Jay Gould. Rhodes does not refer to it by name (it may not have existed when this book was written), but he gives precise quantitative principle concerning "jumps in evolution":

[I] the actual path of evolution of the organism is refined into irreducible jumps, some jumps may double the complexity plus one, but never increase it more than this.

The final section, on Emotion, Neurosis and Schizophrenia, is long and complex. Rhodes introduces a "Lagrangian of individual emotional development", defined in terms of interacting semigroups representing relevant aspects of the individual and the environment, and discusses its relation to neurosis and schizophrenia. In his introduction he summarizes it as follows:

The Lagrangian (true goal) of emotional life for the individual is to contact the environment maximally subject to reasonable understanding and ability to act.

The full audacity, originality, fecundity and rigor of Rhodes' ideas can be appreciated only by reading the book.

\author{
Morris W. Hirsch \\ Department of Mathematics \\ University of California at Berkeley
}


This page intentionally left blank 


\section{Editorial Preface}

"Let there be light!"

- Genesis 1:3

The book you are reading is commonly referred to as the Wild Book. The proof of the Krohn-Rhodes Prime Decomposition Theorem in the 1960s revealed a deep but completely unexpected connection between algebra (semigroups) and computation of all sorts (especially computation requiring only bounded memory). The book in draft manuscript form was first completed by John Rhodes around 1969 and quickly became an underground classic, potentially destined to become the source for fundamental advances in many branches of science. It contains radical ideas on the application of automata and semigroup theory via a rigorously developed theory of mathematical complexity to traditional and non-traditional areas for applications of mathematics. The book introduces a highly insightful approach to create a general, new applied mathematics of finite systems. It is "wild" in that it invades areas as diverse as philosophy (epistemology and the purpose of life), psychology (mathematical theory of psychoanalysis), physics (finite phase-space systems), biology (including metabolism, development and evolution), and games (complexity of games), with a completely original unifying viewpoint and rigorous mathematical methods.

The draft manuscript was used in courses on algebra and automata at the University of California at Berkeley, and was known to students of Rhodes and those lucky enough to find it in the Berkeley Mathematics Library in a simple spiral binding (dated 1971). Many physicists and mathematicians around the world, from Japan to Santa Fe, who became aware of it through academic rumor and word of mouth made photocopies for themselves and disseminated them to colleagues. By the 1990s, many scien- 
tists including Professor Morris Hirsch at Berkeley, who has kindly written the foreword, were encouraging Rhodes to publish the Wild Book. They realized that the approach of introducing sequential coordinates into the understanding of any phenomenon that can be described by a finite-state (or more general) automaton is a fundamental and general contribution to science whose time is coming, even if its full exploitation may require generations of scientists. As with many crucial ideas in mathematics and science that require time to mature, given the depth of these methods and the publication of this book only now even after 47 years since the Krohn-Rhodes Theorem was discovered, it is still only shortly after the first illuminating light-rays of dawn. Let there be light.

Much more has been done and published by John Rhodes and followers of his school, in particular in developing the abstract mathematical theory of finite semigroups and automata, since the first draft of this book was written. Yet due to the limited availability of the material to other scientists and interdisciplinary researchers, most of the suggested applications still need to be developed much further. Applications are also notoriously unfashionable (sometimes even considered scandalous) in the prevalent culture of many pure mathematicians, therefore many of those exposed to and inspired by the material were not being motivated to pursue these aspects. ${ }^{*}$ If nothing else, even casual readers with a traditional view toward applications will likely find their prejudices about the confines of what comprises applied mathematics vigorously shaken by this book, and with just a little further effort may even awaken to substantially broadened horizons of what applied mathematics and applied algebra could look like in the future.

Happily, algorithmic and computational tools implementing Rhodes' ideas in order to automatically generate sequential coordinate systems are finally becoming available to mathematicians and interdisciplinary scientists thanks to the special abilities of a new generation versed not only in the mathematics and its potential application areas, but capable also of efficiently harnessing the computer algebra and high-performance parallel

\footnotetext{
* Indeed, a negative attitude toward applications is common in the orientation of many narrowly enculturated or specialized mathematicians (as the present editor knows only too well from his own intellectual development), but such a viewpoint is either an error of mere prejudice in the prevailing mathematical culture or is based on profound ignorance of the relationship of mathematics to its applications areas. Pure and applied mathematics can drive each other's creative development, as examples from the work of Archimedes, Isaac Newton, John von Neumann, or John Rhodes (e.g. the present volume) make plain.
} 
computation necessary to achieve the full potential of these ideas. ${ }^{\dagger}$ These recent developments are also now approaching the point where some of the techniques described here can be fruitfully applied to natural systems in biology and to artificial intelligence to reveal insights into the hidden algebraic structures and symmetries of these systems in a manner that goes well beyond what is computable by an unaided human being. ${ }^{\ddagger}$

For this first published edition, John Rhodes has written a new philosophical section included here as a prologue, outlining background of the overall viewpoint. Many of the references have been updated in this new edited version of the book while retaining its essential character, errors and typos have been corrected, and an index has been introduced. An almost completely successful effort has been made to retain the numbering of mathematical equations and assertions to agree with the original version. Editorial footnotes are indicated by non-numerical symbols, while the author's own footnotes are indicated with numerals.

Readers of the book, whether from mathematics or other fields, including physicists, biologists of various stripes, computer scientists, psychologists, philosophers, and game theorists, will find much original thought to stimulate the development of their own ideas here. The non-mathematical reader is advised to skip most of the sections developing the mathemati-

\footnotetext{
${ }^{\dagger}$ The first realizations of software implementations of the Krohn-Rhodes Theorem were achieved only at the dawn of the 21st century with the remarkable PhD work of Attila Egri-Nagy at the University of Hertfordshire (see A. Egri-Nagy \& C. L. Nehaniv, "Algebraic Hierarchical Decomposition of Finite State Automata: Comparison of Implementations for Krohn-Rhodes Theory", Springer Lecture Notes in Computer Science 3317:315-316, 2005). Ongoing work is associated with our newer software implementations for mathematical synthesis of hierarchical coordinate systems (i.e. wreath product decompositions usable for prediction, manipulation, understanding and automated solution of problems in finitary discrete dynamical systems). These include Krohn-Rhodes coordinate systems on transformation semigroups (via the holonomy method) and, as a special case, Frobenius-Lagrange coordinate systems on permutation groups. The open-source software (implemented as a package for the GAP computer algebra system) is freely available at http://sourceforge.net/projects/sgpdec/ with some at present minimal documentation.

${ }^{\ddagger}$ For some current and recent work in this direction, the reader is referred to A. EgriNagy \& C. L. Nehaniv, "Hierarchical Coordinate Systems for Understanding Complexity and Its Evolution, with Applications to Genetic Regulatory Networks", Artificial Life (Special Issue on Evolution of Complexity), 14(3):299-312, 2008; A. Egri-Nagy, C. L. Nehaniv, J. L. Rhodes, \& M. J. Schilstra, "Automatic Analysis of Computation in BioChemical Reactions", BioSystems, 94(1-2):126-134, 2008; A. Egri-Nagy \& C. L. Nehaniv, "Algebraic Properties of Automata Associated to Petri Nets and Applications to Computation in Biological Systems", BioSystems, 94(1-2):135-144, 2008; as well as to subsequent and forthcoming papers by these authors and collaborators.
} 
cal theory (Chapters 2, 3 and 5) on first reading and concentrate on the applications sections (the prologue introducing the philosophical view of semigroups as algebraic models of time, the brief overview of the book in Chapter 1, and the very important and deeply insightful Chapter 4 on coordinate systems for understanding phenomena in science, physics, and biochemistry, and then the four substantial Parts of Chapter 6 on metabolism, biology, psychology, and games, with their wide ranging vision and more detailed developments of the viewpoint for various fields). Most of these sections can be understood prior to developing a detailed understanding of the underlying mathematics. Given these applications as motivations, these readers can later pursue the more mathematical chapters. Chapters 2 and 3 explain and justify the research program for finite semigroups from the mathematical viewpoint of finite algebra (especially group theory) and complexity. The motivating material in these chapters introduces a guiding viewpoint on finite semigroup theory as a generalization of finite group theory, with the natural development of the complexity theory framing the study of finite semigroups (and therefore, as the reader shall see in later Chapters, of finite state automata and, therefore, myriad other topics). These parts will be most accessible to those with some background in abstract algebra. Chapter 5 explains the mathematical core of the Krohn-Rhodes prime decomposition theory for finite state machines and then develops the associated complexity theory. The appendix to Chapter 5 describes the connection between the cascade product and to series-parallel product of circuits and provides mathematical proofs of many of the assertions of Chapter 5; this is the most technical section of the book, providing valuable insights for more mathematically sophisticated readers.

The ideas presented in Chapter 6 on applications to biology and psychology (together with their philosophical interpretations), to games, and in Chapter 4 to physics are, for the most part, a still untapped intellectual gold mine for applications of (group-)complexity.

The editor is grateful to the University of Hertfordshire for support and encouragement during the preparation of this volume (especially to Professors Jill Hewitt, Bruce Christianson, John Senior, Kerstin Dautenhahn, and Martin Loomes). In typesetting, copyediting, correcting, putting together this material, and/or indexing, the editor depended on the various skills and invaluable work of Deborah Craig, Attila Egri-Nagy, Laura Morland Rhodes, and the always helpful staff at World Scientific Publishing Co., although they cannot be blamed for any remaining errors, gaffes or inconsistency in style, nor the decision to retain the author's unique 
citation style (against advice to the contrary). Artist Anita Chowdry provided wonderfully appropriate mathematically wild artwork for the front and back covers and page ii. Many thanks to her, and also to Lionel de Rothschild and Najma Kazi for permission to reproduce these exquisite creations! The editor also thanks Dr. Maria Schilstra and again especially Dr. Attila Egri-Nagy, both of the Royal Society / Wolfson Foundation BioComputation Research Laboratory at the University of Hertfordshire, for their advice leading to some technical corrections and improvements. As could have been expected, although it is still far from perfect, the work of editing has taken many years longer than planned. But it would be unfair to delay any further and not make this book available to those with the gifts to pursue its richness, rigor, depth, and to explore and develop the new vistas it opens for science and mathematics.

The material is intellectually demanding, but the rewards will more than repay the effort. Depending on the reader, various bits of the contents and style of this book may come as inspiration, revelation, or shock, as those who have studied with Rhodes will know from his inimitable lectures which hold a special intellectual excitement. In lectures and half-day long café meetings with John Rhodes, many of us as young mathematicians (including $26 \mathrm{PhD}$ students he has seen through) learned to really think creatively for ourselves (wordlessly being expected to fill in gaps as if being instructed by a gentle mathematical Zen master), to drop naive and narrow minded notions in mathematics, to be optimistically curious and adventurous, to lash out with creativity and fearless metaphor in mathematical exploration, to create our own mathematical language and concepts as needed, to come back to discipline and reflective judgment backed up by rigorous proofs, and to begin to see the algebra in everything. ${ }^{\S}$ A similar spirit pervades this book. May it help the reader find illumination!

\section{Chrystopher L. Nehaniv Welwyn Garden City, U.K. First Day of Spring, 20 March 2009}

\footnotetext{
$\S$ Attending at least one lecture by John Rhodes has been suggested as an educational requirement for all Berkeley $\mathrm{PhD}$ students in mathematics. There are many colorful tales to tell about Professor Rhodes' lectures, such as the invention of new Greek letters as needed, fluid co-opting of pictures to denote algebraic operations, proofs presented (and understood by the students) without language, relentless perseverance through calculations and in pushing viewpoints, enlightening breakthrough insights, repeated re-engagement with things already proved yielding new questions and perspectives, unfinished sentences, blank slides, etc., but these stories must be told another time.
} 